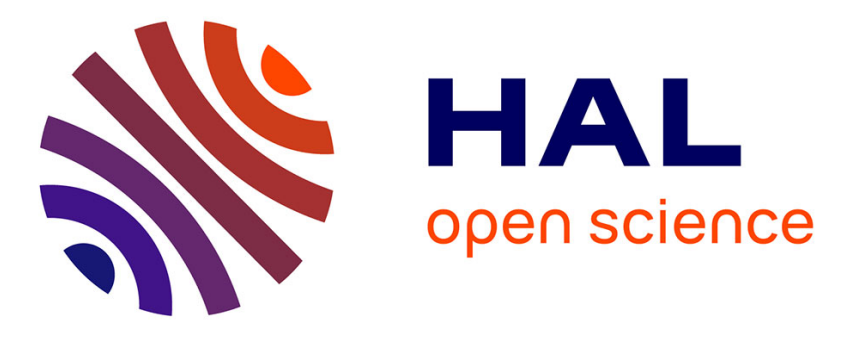

\title{
Adaptive trace-orthonormal STBC for MIMO system with capacity approaching FEC codes
}

\author{
Ammar El Falou, Charlotte Langlais, Charbel Abdel Nour, Catherine \\ Douillard
}

\section{- To cite this version:}

Ammar El Falou, Charlotte Langlais, Charbel Abdel Nour, Catherine Douillard. Adaptive traceorthonormal STBC for MIMO system with capacity approaching FEC codes. VTC2012-Fall 2012: 76th IEEE Vehicular Technology Conference, Sep 2012, Québec, Canada. 10.1109/VTCFall.2012.6399204 . hal-00797517

\section{HAL Id: hal-00797517 https://hal.science/hal-00797517}

Submitted on 23 Jun 2021

HAL is a multi-disciplinary open access archive for the deposit and dissemination of scientific research documents, whether they are published or not. The documents may come from teaching and research institutions in France or abroad, or from public or private research centers.
L'archive ouverte pluridisciplinaire HAL, est destinée au dépôt et à la diffusion de documents scientifiques de niveau recherche, publiés ou non, émanant des établissements d'enseignement et de recherche français ou étrangers, des laboratoires publics ou privés. 


\title{
Adaptive trace-orthonormal STBC for MIMO system with capacity approaching FEC codes
}

\author{
A. El Falou, C. Langlais, C. Abdel Nour and C. Douillard \\ Institut Telecom; Telecom Bretagne; UMR CNRS 3192 Lab-STICC \\ Electronics Department, Technopôle Brest Iroise CS 83818, 29238 Brest Cedex 3 \\ Université européenne de Bretagne, France \\ Email: \{ammar.elfalou, charlotte.langlais, charbel.abdelnour, catherine.douillard $\} @$ telecom-bretagne.eu
}

\begin{abstract}
Space time block codes (STBCs) are commonly designed according to the rank-determinant criteria, suitable for high signal to noise ratio (SNR) values. However, capacity approaching forward error correcting codes, used in practical communication systems, achieve iterative convergence at low to moderate SNR. In this paper we first present a non-asymptotic STBC design criterion based on the bitwise mutual information (BMI) maximization at a specific target SNR. According to this BMI criterion, we then optimize a trace-orthonormal-based STBC structure. Therefore, designed STBC becomes adaptive with respect to the SNR. Proposed adaptive trace-orthonormal STBC shows identical or better performance than $2 \times 2$ STBCs of a turbo-coded WiMAX system.
\end{abstract}

\section{INTRODUCTION}

Multiple input multiple output (MIMO) systems have been adopted in most of the recently developed wireless communication systems. Indeed, they provide higher spectral efficiency (multiplexing gain) and/or better performance (spatial diversity gain) [1] than single input single output (SISO) systems.

In order to design space time block codes (STBCs) for MIMO systems, several design criteria have been previously formulated. Based on the minimization of the pairwise error probability (PEP) between transmitted and detected codewords, the well-known asymptotic rank-determinant criteria aim at designing full-rate full-diversity STBCs [2]. In [3], the linear dispersion STBCs were designed to maximize the symbolwise mutual information between transmit and receive signals. However, these previous works do not take into account the presence of powerful forward error correction (FEC) codes in the transmission chain that usually achieve iterative convergence for low to moderate SNRs. Indeed, in [4], it is stated that uncoded error probability could be misleading, and reveals to be insufficient to predict performance when a STBC is concatenated with an outer powerful FEC code. Moreover, FEC decoding is extremely sensitive to its input bitwise mutual information (BMI) value. Indeed, the higher the $\mathrm{BMI}$ at the input of the FEC decoder, the lower the error rate at the output of the decoder. Our work focuses on the design of a STBC for a practical communication system. The reduction of the end-to-end bit error probability (BER) is targeted. Finally, unlike [5], we focus on low latency communication systems that should avoid the use of iterative MIMO detection.

In this paper, we first present a non-asymptotic MIMO STBC design criterion based on the BMI maximization between transmitted and soft detected bits for a specific target SNR. Without loss of generality, we restrict our study to a $2 \times 2$ MIMO system. According to the rank-determinant criteria, several $2 \times 2$ full-rate full-diversity linear dispersion STBCs have been proposed [6-13]. Among them, the full-rate fulldiversity trace-orthonormal (TO) [6] has a flexible structure due to the presence of a rotation angle that plays the role of a design parameter. Based on the proposed BMI criterion, we compute the appropriate angle maximizing the BMI at the TO output for each SNR. This leads to the proposal of an adaptive TO STBC optimized for a wide range of SNRs. In a communication system using an adaptive modulation and coding like WiMAX, our simulations show that the adaptive TO at least matches all WiMAX $2 \times 2$ MIMO profiles over a quasi-static Rayleigh fading channel.

The remainder of this paper is organized as follows. In Section II, the practical communication MIMO system model under consideration is presented. In Section III, the conventional STBC design criteria and a brief review of well-known $2 \times 2$ MIMO STBC are given. The BMI criterion and the proposed adaptive TO STBC are presented in Section IV. End-to-end BER curves are provided in Section V. Section VI concludes the paper.

\section{System Model AND NOTATIONS}

We consider a MIMO system with $N_{t}$ transmit antennas, $N_{r}$ receive antennas operating over a quasi-static Rayleigh fading channel, i.e., the channel changes for each transmitted MIMO codeword of length $T$. A perfect channel state information (CSI) is assumed only at the receiver. The system is described as follows (see Fig. 1):

Transmitter side: the information word $\mathbf{b}$ is encoded via $\mathbf{a}$ capacity approaching FEC code $\mathrm{C}$ with rate $R_{c}$. The resulting codeword is interleaved with a random interleaver $\Pi$ following a bit interleaved coded modulation (BICM) scheme [14]. We denote by $\mathbf{c}$ the interleaved codeword. $\mathbf{c}$ is mapped onto a Gray encoded $M$-QAM constellation where $M=2^{m}$ is the modulation order and $m$ denotes the number of bits per symbol. A MIMO system is said to be full-rate (FR) when the number of transmitted symbols per channel use is equal to the number of transmit antennas $N_{t}$. The mapper feeds a block of $N_{t} \times T$ QAM symbols denoted by $S_{i}$ to the STBC MIMO 


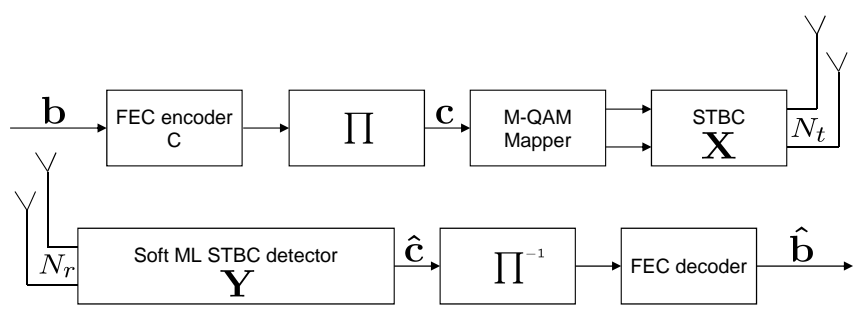

Fig. 1. Structure of the system at the transmitter and the receiver.

encoder. Afterwards, encoder output denoted by $\mathbf{X}_{\left[N_{t} \times T\right]}$ is transmitted via $N_{t}$ antennas over $T$ channel use periods.

The MIMO channel is completely defined by the matrix $\mathbf{H}_{\left[N_{r} \times N_{t}\right]}$ where its entries are assumed to be independent and identically distributed (i.i.d.) circularly symmetric Gaussian random variables with probability density function (pdf) $\sim C N(0,1) . C N\left(\mu, \sigma^{2}\right)$ denotes the complex Gaussian pdf with mean $\mu$ and variance $\sigma^{2}$. A complex white Gaussian noise $\mathbf{N}_{\left[N_{r} \times T\right]}$ with i.i.d. entries and pdf $\sim C N\left(0, \sigma^{2}\right)$ is added to the received signals.

The channel input-output relation is be given by:

$$
\mathbf{Y}=\mathbf{H X}+\mathbf{N}
$$

Receiver side: $N_{r} \times T$ noisy signals, denoted by $\mathbf{Y}_{\left[N_{r} \times T\right]}$, are received on the $N_{r}$ antennas. A soft maximum-likelihood (ML) STBC detector estimates the transmitted $M$-QAM symbols and feeds the $P\left(c_{i}=1\right)\left(c_{i}\right.$ is the $i$-th bit of codeword c), denoted by $\hat{c}_{i}$, to the deinterleaver. These probabilities are deinterleaved then provided to the FEC decoder. Resulting hard decoded information word $\hat{b}$ is compared to $\mathbf{b}$ to compute the BER.

\section{STATE OF THE ART: $2 \times 2$ MIMO STBCS}

\section{A. Conventional STBC design criteria}

Minimizing the STBC codeword error probability or equivalently minimizing the union bound for the PEP leads to the well-known rank-determinant STBC design criteria [2]:

1) Rank Criterion: To achieve the maximum diversity, the rank $r$ of the codeword difference matrix $\Delta=\mathbf{X}-\hat{\mathbf{X}}$ must be maximized for all possible transmitted codeword pairs $(\mathbf{X}, \hat{\mathbf{X}})$. The diversity gain is defined as $d=r N_{r}$. When $r=N_{t}$ the system is said to be full-diversity (FD).

2) Determinant Criterion: The STBC minimum determinant is defined as:

$$
\delta=\min _{\mathbf{X} \neq \hat{\mathbf{X}}} \prod_{i=1}^{r} \lambda_{i}
$$

where $\lambda_{i} ; i=1, \ldots, r$ are the non-zero eigenvalues of the matrix $\Delta \Delta^{H}\left(\Delta^{H}\right.$ is the Hermitian of $\left.\Delta\right)$.

In order to obtain the best performance at high SNR values, $\delta$ should be maximized. Indeed, the dominant parameter is the diversity gain $d$ which defines the slope of BER curves. Therefore, it is important to ensure the FD of the STBC and then maximize its coding gain $\delta^{1 / N_{t}}$.

\begin{tabular}{|c|c|}
\hline $\begin{array}{c}\text { MIMO Code } \\
\mathbf{X}\end{array}$ & $\begin{array}{c}\text { Minimum determinant } \\
\text { for QAM modulations }\end{array}$ \\
\hline \hline Golden code (GC) [7] & 3.2000 \\
\hline Srinath-Rajan (SR) [9] & 3.2000 \\
\hline Dayal-Varanasi (DV) [8] & 3.2000 \\
\hline Trace-orthonormal (TO) [6] & 3.2000 \\
\hline HTW-PGA [12, 13] & 2.2857 \\
\hline Sezginar-Sari (MD) [11] & 2.0000 \\
\hline Yao-Wornell (YW) [10] & 0.8000 \\
\hline Spatial multiplexing (SM) [17] & 0.0000 \\
\hline
\end{tabular}

TABLE I

COMPARISON BETWEEN THE MINIMUM DETERMINANT OF SOME WELL-KNOWN FULL-RATE $2 \times 2$ STBCS.

\section{B. WiMAX $2 \times 2$ MIMO profiles}

The worldwide interoperability for microwave access (WiMAX) system uses the MIMO codes specified in the IEEE 802.16e-2005 standard [15]. Two mandatory MIMO profiles are described for the downlink. The first represents the Alamouti code [16] introduced for transmit diversity, referred to as Matrix A. It offers FD but is only half-rate. The second profile is the FR spatial multiplexing (SM) [17] introduced for spectral efficiency increase, referred to as Matrix B. In order to benefit from both diversity and multiplexing gains, another MIMO profile is included in the IEEE 802.16e-2005 specification, referred to as Matrix $\mathrm{C}$ which is a variant of the Golden code (GC) [7]. At high SNRs, the GC is known as the best $2 \times 2$ FR-FD STBC with non-vanishing determinants.

\section{STBCs for $2 \times 2$ MIMO system}

For the $2 \times 2$ MIMO system, several linear dispersion FR-FD STBCs have been proposed in [6-13]. These codes are defined under the rank-determinant criteria. Table I summarizes their characteristics. The minimum determinant is computed for symbols chosen from a regular $M$-QAM constellation where the difference between any two constellation points is a multiple of 2 .

Among them, the TO STBC has a flexible structure designed from both information-theoretic and detection error viewpoints [6]. By varying one of the code parameters, the performance of the TO STBC varies significantly. Therefore we propose to take advantage of this feature in order to design a flexible TO-based STBC. The resulting structure is intended to at least match and in some cases overcome existing STBCs for a wide range of SNRs.

\section{ADAPTIVE TRACE-ORTHONORMAL STBC}

In this section, we first present the bitwise mutual information criterion. Then, we apply this criterion to the TO STBC to design an adaptive STBC with respect to a wide range of SNR values.

\section{A. BMI criterion}

The asymptotic rank-determinant criteria optimize STBCs for high SNRs. Most of practical communication systems are 


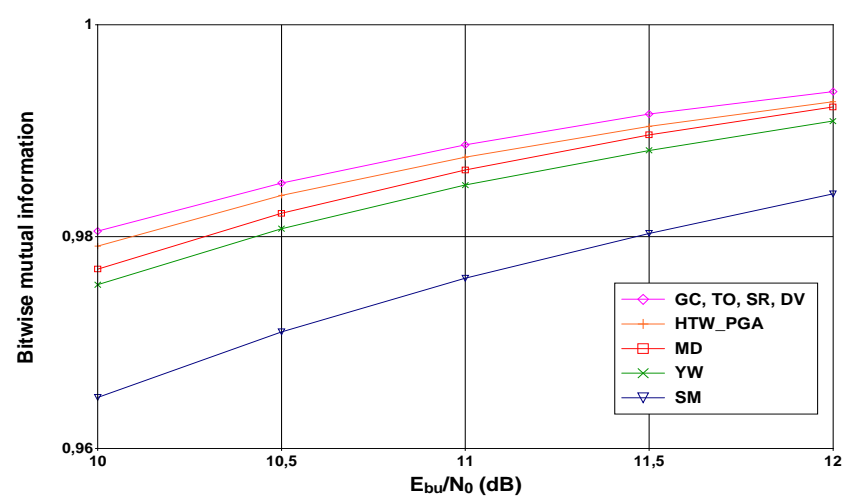

Fig. 2. BMI of some well-known $2 \times 2$ MIMO STBCs for high $E_{b u} / N_{0}$; 4-QAM modulation.

planned for low or moderate target SNRs due to the introduction of a capacity approaching FEC code. Therefore, the STBC parameter optimization should be targeted for this SNR range. Based on the maximization of the BMI between transmitted and soft detected bits, we propose a non-asymptotic design criterion aiming at choosing the appropriate design parameter value for STBCs at a specific target SNR.

The BMI is computed as in [18], by:

$$
\begin{array}{r}
\operatorname{BMI}(\hat{c} ; c)=1-E\left[\log _{2}(1+\exp (-L))\right] \\
\approx 1-\frac{1}{N} \sum_{n=1}^{N} \log _{2}\left(1+\exp \left(-u_{n} . L_{n}\right)\right) \\
\text { with }\left\{\begin{array}{l}
L_{n}=\ln \frac{1-\hat{c}_{n}}{\hat{c}_{n}} \\
u_{n}=(-1)^{c_{n}}
\end{array}\right.
\end{array}
$$

where $E$ is the mean function and $L$ denotes the negative loglikelihood ratio (NLLR). $N$ is assumed to be large enough to accurately estimate the BMI. The BMI value is assessed by Monte Carlo simulations by passing a $N$-bit sequence into the mapper, the STBC, the MIMO channel and the soft ML detector. In this work, we considered $N=10^{7}$ simulated bits.

In order to validate the BMI criterion from a MIMO coding gain point of view, we have plotted in Fig. 2 the BMI of wellknown $2 \times 2$ MIMO codes presented in Table I, for a 4-QAM modulation, as a function of high $E_{b u} / N_{0}$ values, where $E_{b u}$ denotes the energy per bit for uncoded MIMO system and $N_{0}$ the noise power spectral density. We use the expression "uncoded" to refer to the case of a MIMO STBC system without any FEC code. STBCs in $[6,8,9]$ i.e., DV, TO and SR show a quasi identical performance than the GC. Fig. 2 shows that the hierarchy of the presented MIMO codes is identical to their classification in Table I.

Although we have restricted our study to the $2 \times 2$ TO STBC, the application of the BMI criterion to any $N_{t} \times N_{r}$ STBC is straightforward.

\section{B. Optimization of trace-orthonormal STBC}

We apply the BMI criterion to the trace-orthonormal linear dispersion FR-FD STBC presented in [6].

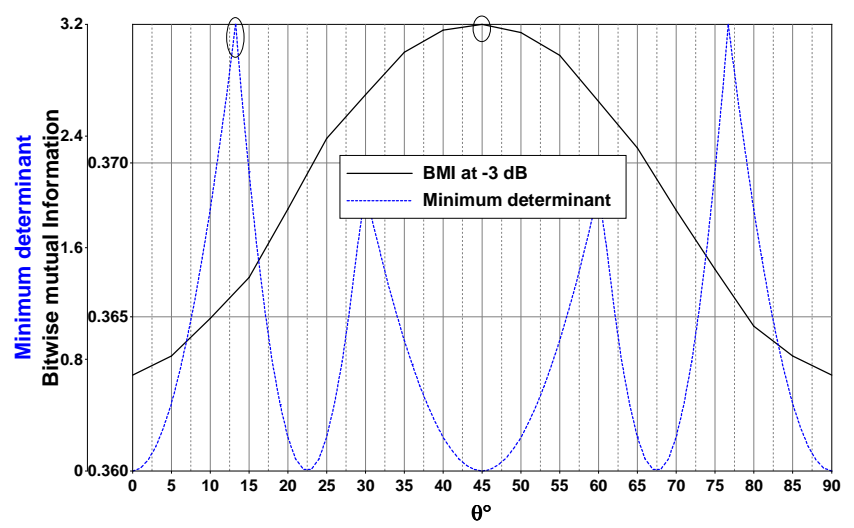

Fig. 3. BMI at $E_{b u} / N_{0}=-3 \mathrm{~dB}$ and minimum determinant of TO STBC as a function of its design parameter $\theta ; 4$-QAM modulation.

A group of 4 data symbols $\left(S_{1}, S_{2}, S_{3}, S_{4}\right)$ is transmitted as follows:

$$
\mathbf{X}^{\mathrm{TO}}=\frac{1}{\sqrt{2}}\left[\begin{array}{ll}
X_{11} & X_{12} \\
X_{21} & X_{22}
\end{array}\right]
$$

where

$$
\begin{aligned}
& X_{11}=\left(S_{1}+S_{2}\right) \cos \theta+\left(S_{2}^{*}-S_{1}^{*}\right) \sin \theta \\
& X_{12}=e^{\frac{j \pi}{4}}\left(\left(S_{3}+S_{4}\right) \sin \theta+\left(S_{4}^{*}-S_{3}^{*}\right) \cos \theta\right) \\
& X_{21}=e^{\frac{j \pi}{4}}\left(\left(S_{3}+S_{4}\right) \cos \theta+\left(S_{3}^{*}-S_{4}^{*}\right) \sin \theta\right) \\
& X_{22}=\left(S_{1}+S_{2}\right) \sin \theta+\left(S_{1}^{*}-S_{2}^{*}\right) \cos \theta
\end{aligned}
$$

$S^{*}$ designates the complex conjugate of $S$ and $\theta$ is the code design parameter to be optimized according to the selected criterion.

In [6], an exhaustive search is performed in order to maximize the minimum determinant. This search leads to a $\theta=\frac{1}{2} \arcsin \frac{1}{\sqrt{5}} \approx 13.28^{\circ}$ and a coding gain equal to the one of the Golden code. Therefore, original TO guarantees a good performance for high SNRs, but not necessary for low and moderate SNRs.

In Fig. 3 and Fig. 4, the minimum determinant and the BMI are plotted as a function of the design parameter $\theta$, for a 4-QAM modulation, for $E_{b u} / N_{0}=-3 \mathrm{~dB}$ and $E_{b u} / N_{0}=$ $12 \mathrm{~dB}$ respectively. For low $E_{b u} / N_{0}$, Fig. 3 shows that the angle which maximizes the BMI is equal to $\theta=45^{\circ}$, different from the original one obtained under the determinant criterion. While for high $E_{b u} / N_{0}$ (see Fig. 4), the obtained $\theta$ under the BMI criterion is equal to $13.28^{\circ}$ as the original one obtained in [6]. These results show that the TO design parameter value is not unique for both low and high SNRs. As presented in Fig. 3 and Fig. 4 for $E_{b u} / N_{0}=-3 \mathrm{~dB}$ and $E_{b u} / N_{0}=12 \mathrm{~dB}$ respectively, we have computed for each $E_{b u} / N_{0}$ with a step of $0.25 \mathrm{~dB}$, the appropriate $\theta$ which maximizes its BMI. We denote the obtained $\theta$ by $\theta_{\mathrm{opt}}^{M-\mathrm{QAM}}$.

For 4-QAM modulation, in the range of $-0.5 \mathrm{~dB}<$ $E_{b u} / N_{0}<4.25 \mathrm{~dB}$, a $\theta_{\mathrm{opt}}^{4-\mathrm{QAM}}$ is obtained for each $E_{b u} / N_{0}$. A polynomial interpolation is performed to obtain an analytical approximation of $\theta_{\mathrm{opt}}^{4-\mathrm{QAM}}$ as a function of $E_{b u} / N_{0}$. Therefore, the design parameter of the proposed adaptive TO STBC which 


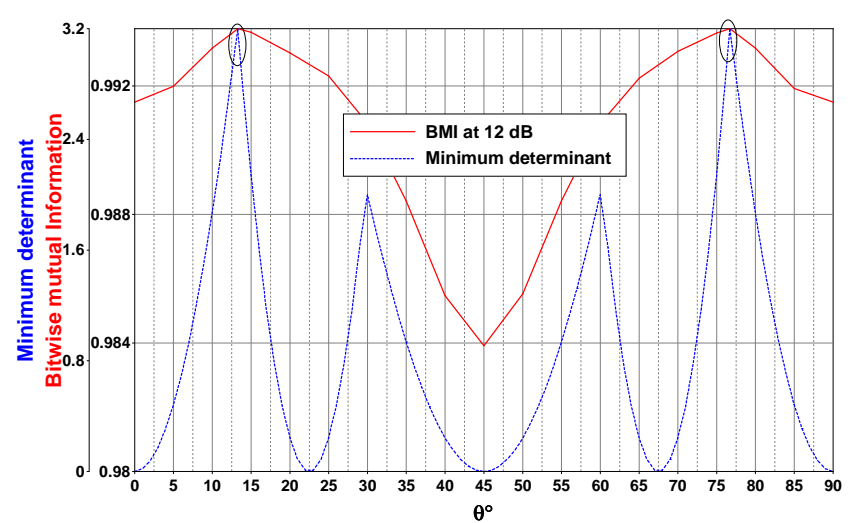

Fig. 4. BMI at $E_{b u} / N_{0}=12 \mathrm{~dB}$ and minimum determinant of TO STBC as a function of its design parameter $\theta ; 4$-QAM modulation.

maximizes its BMI is computed, for all SNRs, by:

$$
\theta_{\mathrm{opt}}^{4-\mathrm{QAM}}=\left\{\begin{array}{c}
45^{\circ} ; \text { For } E_{b u} / N_{0} \leq-0.5 \mathrm{~dB} \\
-0.65\left(\frac{E_{b u}}{N_{0}}\right)^{3}+4.79\left(\frac{E_{b u}}{N_{0}}\right)^{2}-13.8\left(\frac{E_{b u}}{N_{0}}\right) \\
+36.47 ; \text { For }-0.5<E_{b u} / N_{0}<4.25 \mathrm{~dB} \\
13.28^{\circ} ; \text { For } E_{b u} / N_{0} \geq 4.25 \mathrm{~dB}
\end{array}\right.
$$

Similarly, for 16-QAM modulation, the design parameter $\theta_{\mathrm{opt}}^{16-\mathrm{QAM}}$ is computed by:

$$
\theta_{\mathrm{opt}}^{16-\mathrm{QAM}}=\left\{\begin{array}{c}
45^{\circ} ; \text { For } E_{b u} / N_{0} \leq 9 \mathrm{~dB} \\
0.424\left(\frac{E_{b u}}{N_{0}}\right)^{2}-14.936\left(\frac{E_{b u}}{N_{0}}\right) \\
+139 ; \text { For } 9<E_{b u} / N_{0}<13.5 \mathrm{~dB} \\
13.28^{\circ} ; \text { For } E_{b u} / N_{0} \geq 13.5 \mathrm{~dB}
\end{array}\right.
$$

For higher order modulations, the same method can be applied and a suitable $\theta_{\mathrm{opt}}^{M-\mathrm{QAM}}$ can be chosen. The BMI criterion always provides the best choice for the TO design parameter since it maximizes the BMI for all SNRs. We notice that an analytical method to get $\theta_{\mathrm{opt}}^{M-\mathrm{QAM}}$ is still an open problem.

\section{ML detection complexity of adaptive TO STBC}

Practical communication systems promote the usage of low complexity detection STBCs. In the range of low $E_{b u} / N_{0}$, the optimized TO parameter is $\theta_{\mathrm{opt}}^{M-\mathrm{QAM}}=45^{\circ}$ (see Section IV-B). If we consider:

$$
\begin{aligned}
& S_{1}^{\prime}=\Re\left(S_{2}\right)+j \Im\left(S_{1}\right) \\
& S_{2}^{\prime}=\Re\left(S_{3}\right)+j \Im\left(S_{4}\right) \\
& S_{3}^{\prime}=\Re\left(S_{4}\right)+j \Im\left(S_{3}\right) \\
& S_{4}^{\prime}=\Re\left(S_{1}\right)+j \Im\left(S_{2}\right)
\end{aligned}
$$

The TO STBC for a $\theta=45^{\circ}$ reduces to:

$$
\mathbf{X}_{\theta=45^{\circ}}^{\mathrm{TO}}=\frac{1}{\sqrt{2}}\left[\begin{array}{cc}
S_{1}^{\prime} & e^{\frac{j \pi}{4}} S_{3}^{\prime} \\
e^{\frac{j \pi}{4}} S_{2}^{\prime} & S_{4}^{\prime}
\end{array}\right]
$$

$\left(S_{1}^{\prime}, S_{2}^{\prime}, S_{3}^{\prime}, S_{4}^{\prime}\right)$ are $M$-QAM symbols. Therefore, $\left(S_{1}^{\prime}, S_{2}^{\prime}\right)$ can be detected with a SM detector of complexity $\mathcal{O}\left(M^{2}\right)$ since their detection is independent from $\left(S_{3}^{\prime}, S_{4}^{\prime}\right)$. Similarly, $\left(S_{3}^{\prime}, S_{4}^{\prime}\right)$ detection is independent from $\left(S_{1}^{\prime}, S_{2}^{\prime}\right)$. Then, the

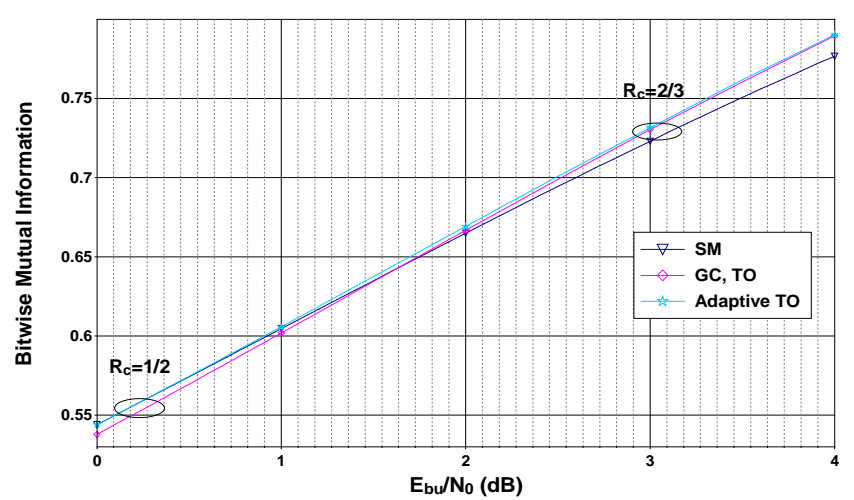

Fig. 5. BMI of WiMAX MIMO profiles and adaptive TO in the region of interest as a function of $E_{b u} / N_{0} ; 4$-QAM modulation.

original symbols can be easily reconstructed by:

$$
\begin{aligned}
& S_{1}=\Re\left(S_{4}^{\prime}\right)+j \Im\left(S_{1}^{\prime}\right) \\
& S_{2}=\Re\left(S_{1}^{\prime}\right)+j \Im\left(S_{4}^{\prime}\right) \\
& S_{3}=\Re\left(S_{2}^{\prime}\right)+j \Im\left(S_{3}^{\prime}\right) \\
& S_{4}=\Re\left(S_{3}^{\prime}\right)+j \Im\left(S_{2}^{\prime}\right)
\end{aligned}
$$

However, the ML complexity detection of the GC and the original TO is $\mathcal{O}\left(M^{4}\right)$ [9]. Therefore, the adaptive TO STBC offers identical or better performance than the different $2 \times 2$ STBCs with reduced detection complexity in the range of low $E_{b u} / N_{0}$.

\section{Simulation Results}

\section{A. Adaptive TO parameter selection}

For the WiMAX system, the used FEC code is a 8-state double binary turbo code. In our simulations, such a code is used with different rates $R_{c}=1 / 2,2 / 3$ and $3 / 4$ and an information frame size of 10,800 bits. For the adaptive TO STBC, the appropriate $\theta_{\mathrm{opt}}^{M \text {-QAM }}$ depends on the coding rate $R_{c}$ and the modulation order $M$. This $\theta_{\mathrm{opt}}^{M \text {-QAM }}$ value is computed as follows: 1) Passing from global system to uncoded MIMO by: $E_{b u} / N_{0}=E_{b} / N_{0}+10 \log _{10}\left(R_{c}\right)$ where $E_{b}$ is the energy per information bit. 2) The obtained $E_{b u} / N_{0}$ is introduced in equation (6) and (7) to compute the value of $\theta$ to be used for the proposed TO structure.

Fig. 5 illustrates the BMI of the SM, the GC (and hence the original TO) and the adaptive TO as a function of $E_{b u} / N_{0}$ for a 4-QAM modulation. It shows that the adaptive TO offers the highest BMI of all compared codes. For $R_{c}=1 / 2$, the FEC converges for $3.1 \mathrm{~dB}<E_{b} / N_{0}<3.4 \mathrm{~dB}$ corresponding to $0.1 \mathrm{~dB}<E_{b u} / N_{0}<0.4 \mathrm{~dB}$. Therefore in the $R_{c}=1 / 2$ range, the $\mathrm{SM}$ and the adaptive $\mathrm{TO}$ are equivalent while outperforming the GC. For $R_{c}=2 / 3$, the adaptive TO is slightly better than the GC both outperforming the SM. For $R_{c}=3 / 4$, the GC and the adaptive TO offer the highest BMI.

\section{B. BER curves}

Fig. 6 plots the BER after 15 turbo-decoding iterations as a function of $E_{b} / N_{0}$ for a transmission over a quasi-static Rayleigh fading MIMO channel. A random BICM interleaver 


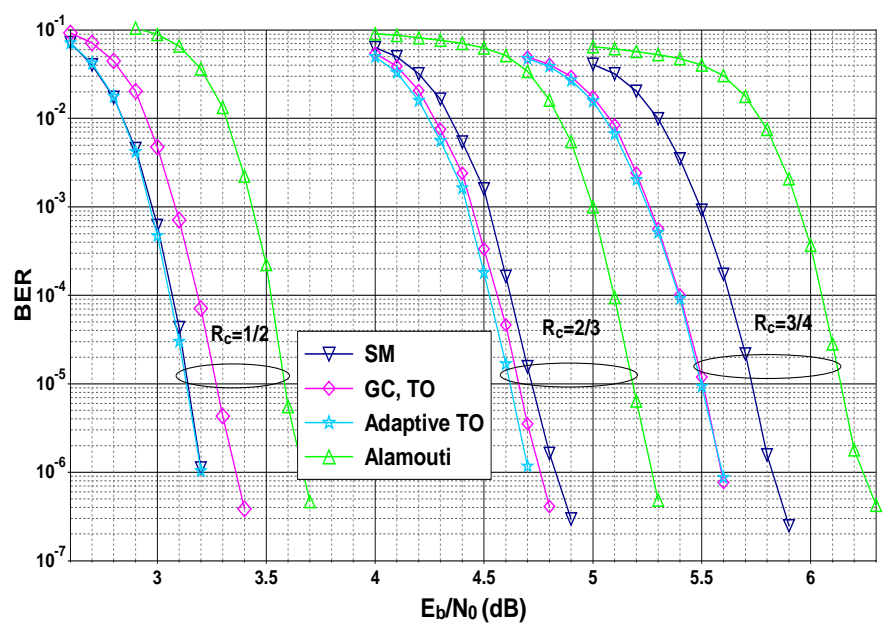

Fig. 6. BER of turbo coded MIMO system; 8-state double binary TC ( $\left.R_{c}=1 / 2,2 / 3,3 / 4\right)$ with 15 turbo-decoding iterations; MIMO profiles: SM, GC\&TO, adaptive TO with 4-QAM and Alamouti with 16-QAM; quasistatic Rayleigh fading MIMO channel.

is used. The number of transmit antennas is $N_{t}=2$ and receive antennas is $N_{r}=2$.

A fair comparison between presented MIMO codes and the Alamouti code should be done at the same spectral efficiency. Therefore, we use a 16-QAM modulation for Alamouti and a 4-QAM modulation for the FR profiles. The BER simulations confirm the results obtained using the BMI criterion. Indeed, the hierarchy in the sense of BMI of the compared STBCs is respected. We evaluate the gains of the adaptive TO at a target $\mathrm{BER}=10^{-5}$. For $R_{c}=1 / 2$, the $\mathrm{SM}$ matches the adaptive $\mathrm{TO}$, a gain of almost $0.15 \mathrm{~dB}$ is obtained with respect to the GC (and hence the original TO) and $0.45 \mathrm{~dB}$ with respect to Alamouti. For $R_{c}=2 / 3$, the adaptive TO surpasses all the presented codes, with a gain of $0.05 \mathrm{~dB}$ with respect to the $\mathrm{GC}, 0.1 \mathrm{~dB}$ with respect to the $\mathrm{SM}$ and $0.55 \mathrm{~dB}$ with respect to Alamouti. For $R_{c}=3 / 4$, the GC matches the adaptive TO, while a gain of $0.2 \mathrm{~dB}$ is observed compared to the SM and $0.65 \mathrm{~dB}$ compared to Alamouti.

For 16-QAM modulation, BER curves show the superiority of the adaptive TO STBC. Their curves are not plotted due to lack of space. We note that the adaptive TO also at least matches the codes presented in Table I.

BER simulation results confirm that the adaptive TO based on the proposed angle $\theta_{\mathrm{opt}}^{M-\mathrm{QAM}}$ outperforms all STBC profiles of the WiMAX system. This is particularly of interest when an adaptive modulation and coding (AMC) is used as the appropriate angles are chosen for each rate. Moreover, the usage of a non-adaptive MIMO code i.e., SM, GC or Alamouti introduces a performance loss for some AMC schemes.

\section{CONCLUSIONS}

In this paper, after summarizing the features of conventional $2 \times 2$ STBCs, we have presented a non-asymptotic design criterion for MIMO STBC based on the BMI maximization at a specific target SNR. Under the proposed criterion, we have optimized the rotation angle of the $2 \times 2$ TO STBC [6] for a wide range of SNRs. Moreover, the adaptive TO detection complexity can be reduced to $\mathcal{O}\left(M^{2}\right)$ when it offers the SM performance. The proposed optimization is important for practical systems especially those with AMC as the appropriate design parameter for the TO STBC is used with each rate. In the context of a WiMAX system, the proposed adaptive TO STBC outperforms or matches the existing MIMO profiles for all rates. On the contrary, the use of a non-adaptive STBC like the well-known Golden code [7], will increase the MIMO detection complexity and alter the performance of terminals for low coding rate profiles.

\section{REFERENCES}

[1] L. Zheng and D. N. C. Tse, "Diversity and multiplexing: a fundamental tradeoff in multiple-antenna channels," IEEE Trans. Inf. Theory, vol. 49, no. 5, pp. 1073-1096, May 2003.

[2] V. Tarokh, N. Seshadri, and A. R. Calderbank, "Space-time codes for high data rate wireless communications: Performance criterion and code construction," IEEE Trans. Inf. Theory, vol. 44, no. 2, pp. 744-765, Mar. 1998.

[3] B. Hassibi and B. M. Hochwald, "High-rate codes that are linear in space and time," IEEE Trans. Inf. Theory, vol. 48, no. 7, pp. 1804-1824, Jul. 2002.

[4] A. Lozano and N. Jindal, "Transmit diversity vs. spatial multiplexing in modern mimo systems," IEEE Trans. Wireless Commun., vol. 9, no. 1, pp. 186 - 197, Jan. 2010.

[5] N. Gresset, L. Brunel, and J. J. Boutros, "Space-time coding techniques with bit-interleaved coded modulations for MIMO block-fading channels," IEEE Trans. Inf. Theory, vol. 54, no. 5, pp. 2156-2178, May 2008.

[6] J.-K. Zhang, J. Liu, and K. M. Wong, "Trace-orthonormal full-diversity cyclotomic space-time codes," IEEE Trans. Signal Processing, vol. 55, no. 2, pp. 618-630, Feb. 2007.

[7] J.-C. Belfiore, G. Rekaya, and E. Viterbo, "The golden code: a $2 \times 2$ full-rate space-time code with nonvanishing determinants," IEEE Trans. Inf. Theory, vol. 51, no. 4, pp. 1432-1436, Apr. 2005.

[8] P. Dayal and M. K. Varanasi, "An optimal two transmit antenna spacetime code and its stacked extensions," IEEE Trans. Inf. Theory, vol. 51, no. 12 , pp. 4348-4355, Dec. 2005.

[9] K. P. Srinath and B. S. Rajan, "A low-complexity, full-rate, full-diversity $2 \times 2$ STBC with golden code's coding gain," in Proc. IEEE Global Telecommun. Conf. (GLOBECOM), Dec. 2008.

[10] H. Yao and G. W. Wornell, "Achieving the full MIMO diversitymultiplexing frontier with rotation-based space-time codes," in Proc. Allerton Conf. Comm. Control and Computing, Oct. 2003.

[11] S. Sezginer and H. Sari, "Full-rate full-diversity $2 \times 2$ space-time codes of reduced decoder complexity," IEEE Commun. Lett., vol. 11, no. 12, pp. 973-975, Dec. 2007.

[12] J. M. Paredes, A. B. Gershman, and M. Gharavi-Alkhansari, "A new fullrate full-diversity space-time block code with nonvanishing determinants and simplified maximum-likelihood decoding," IEEE Trans. Signal Processing, vol. 56, no. 6, pp. 2461-2469, Jun. 2008.

[13] A. Hottinen, O. Tirkkonen, and R. Wichman, Multi-antenna Transceiver Techniques for $3 G$ and Beyond. John Wiley \& Sons, 2003.

[14] G. Caire, G. Taricco, and E. Biglieri, "Bit-interleaved coded modulation,” IEEE Trans. Inf. Theory, vol. 44, no. 3, pp. 927 -946, May 1998.

[15] "IEEE 802.16-2005: IEEE standard for local and metropolitan area networks - Part 16: Air Interface for Fixed and Mobile Broadband Wireless Access Systems - Amendment 2: Physical Layer and Medium Access Control Layers for Combined Fixed and Mobile Operation in Licensed Bands," Feb. 2006.

[16] S. M. Alamouti, "A simple transmit diversity technique for wireless communications," IEEE J. Sel. Areas in Commun., vol. 16, no. 8, pp. 1451-1458, Oct. 1998.

[17] P. W. Wolniansky, G. J. Foschini, G. D. Golden, and R. A. Valenzuela, "V-blast: an architecture for realizing very high data rates over the rich-scattering wireless channel," in Proc. IEEE Int. Symp. on Signals, Syst. and Electronics (ISSSE), Sep. 1998.

[18] J. Hagenauer, "The EXIT chart-introduction to EXtrinsic Information Transfer in iterative processing," in Proc. European Signal Processing Conf., Sep. 2004, pp. 1541-1548. 